\title{
A MULTI-CENTRE CLINICAL EVALUATION OF ISOFLURANE
}

\author{
JAMES B. ForRest \\ Professor \& Chairman, Department of Anaesthesia, McMaster University, Hamilton, Ontario, Canada. \\ Charles Buffington \\ Assistant Professor, Department of Anesthesiology, University of Washington, Seatlle, Washington. \\ Michael K. Cahalan \\ Assistant Professor, Department of Anaesthesia, University of California San Francisco, San Francisco, California. \\ Charles H. Goldsmith \\ Professor, Epidemiology \& Biostatistics, McMaster University, Hamilton, Ontario, Canada. \\ WARREN LEVY \\ Assistant Professor, Dept. of Anesthesia, University of Pennsylvania, Philadelphia, Pennsylvania.
}

KaI ReHDER

Professor, Department of Anesthesiology, Maya Clinic, Rochester, Minnesota

\section{INTRODUCTION}

IsoFLURANE (1-chloro-2,2,2-trifluoroethyl difluroromethyl ether) was synthesized in 1965 by R.C. Terrell two years after development of its isomer, enflurane. It is nonflammable at all concentrations used for clinical anaesthesia, is stable without the need for preservatives and is not degraded during five years of storage. It has a molecular weight of 184.5 , a specific gravity of 1.50 at $20^{\circ} \mathrm{C}$, a boiling point of $48.5^{\circ} \mathrm{C}$ and vapour pressure at $20^{\circ} \mathrm{C}$ of $31.85 \mathrm{kPa}(239.5$ torr) compared to $32.46 \mathrm{kPa}$ (244.1 torr) for halothane. 1

Clinical trials in human volunteers confirmed the rapid uptake of isoflurane (blood/gas solubility coefficient equals 1.4 ) and equally rapid recovery with minimal postanaesthetic sequelae. Metabolism of isoflurane was shown to be minimal while its relaxant properties, including potentiation of muscle relaxant drugs, were relatively potent. It produces peripheral vascular vasodilation and hypotension but does not alter cardiac output and is the least arrhythmogenic anaesthetic currently available. ${ }^{2}$

The introduction of isoflurane into clinical practice was originally scheduled for 1975; however a small pilot study by Corbett ${ }^{3}$ in mice

Address for correspondence: J.B. Forrest, M.D., Ph.D., Professor \& Chairman, Department of Anaesthesia, McMaster University, Hamilton, Ontario, Canada L8N 3Z5.

Can. Anaesth. Soc. J., vol. 29, Supplement 1982 suggested that it might be hepatocarcinogenic. Extensive animal studies were therefore conducted using a wider range of isoflurane concentrations in a more controlled manner. These studies failed to show any evidence of carcinogenicity. ${ }^{4}$ Subsequent examination of the conditions under which Corbett conducted his studies revealed that these mice had been contaminated with polybrominated biphenyls, which are known potent teratogens and mutagenic carcinogens. In 1980 isoflurane was approved for general clinical use by the Food and Drug Administration in the United States and by the Health Protection Branch of the Ministry of Health in Canada.

As a preliminary to its widespread introduction into clinical practice a multi-centre clinical evaluation of isoflurane was conducted in 165 university teaching hospitals throughout Canada and the United States. A standardized data collection form was used to document demographic information, pulse and blood pressure changes, the concentrations of isoflurane used and to record in detail the incidence of arrhythmias, reflex activity and complications.

This report details the findings of an extensive clinical evaluation of isoflurane administered to a total of 7,196 patients and represents one of the largest multi-centre new drug studies ever undertaken. The participating institutions and study monitors are listed separately in recognition of their efforts and cooperation without which this study would not have been possible. 